Powerful sequential designs using Bayesian estimation: A power analysis tutorial using brms, the tidyverse, and furrr

James W. B. Elsey

Author Note

Department of Clinical Psychology, University of Amsterdam, Amsterdam, Netherlands

This manuscript was supported by Prof. Dr Merel Kindt's ERC Advanced Grant 743263.

James Elsey has no conflicts of interest to declare. Code and data for this tutorial are available at: https://osf.io/32uxa/?view_only=d8930640e62e492b96d5d2da9eb8839c 


\begin{abstract}
Producing compelling and trustworthy results relies upon performing well-powered studies with low rates of misleading evidence. Yet, resources are limited, and maximum sample sizes required to achieve acceptable power in typical fixed $N$ designs may be disconcerting. 'Sequential', 'optional stopping', or 'interim' designs - in which results may be checked at interim periods and a decision made as to whether to continue data collection or not - provide one means by which researchers may be able to achieve high power and low false positive rates with less of a resource burden. Sequential analyses have received considerable attention from both frequentist and Bayesian hypothesis testing approaches, but fewer approachable resources are available for those wishing to use Bayesian estimation. In this tutorial, we cover a general process for performing power analyses of fixed and sequential designs using Bayesian estimation - simulating data, performing regressions in parallel to reduce time requirements, choosing different stopping criteria and data collection sequences, and calculating observed power and rates of misleading evidence. We conclude with a discussion of some limitations and possible extensions of the presented approach.
\end{abstract}

Keywords: Bayesian estimation; power analysis, brms; sequential analysis; optional stopping 


\section{Powerful sequential designs using Bayesian estimation: A power analysis tutorial using brms, the tidyverse, and furrr}

Designing studies so that one has a reasonable likelihood of detecting an effect where it exists is an important - even crucial - part of the research process. Yet, underpowered studies are common in psychological and other sciences (Lamberink et al., 2018; Szucs \& Ioannidis, 2017). When studies are underpowered, we run several risks. We may miss important effects that really exist because our studies are not sufficiently powered to detect them. Effects that we do observe are also more likely to be overestimates of the population level effect in underpowered vs. well-powered designs, because abnormally extreme results may be the only ones a study is able to detect (Ioannidis, 2005). Hence, observed effects may be inflations of the true effect or even false positives - a problem which is exacerbated by selective reporting of 'significant' effects. In short, poorly powered designs may increase both false positive and false negative rates, with detrimental effects on the growth of knowledge about important psychological phenomena.

Power analysis may be relatively neglected by researchers for several reasons. Firstly, it requires learning how to perform and interpret power analyses for the specific test one wishes to perform. Although a range of software has been developed for this purpose, power analyses are not always easy. Perhaps more importantly, power analyses can suggest frustratingly large sample size requirements, especially relative to some simple rules of thumb for sample sizes that are reported to be pervasive (cf. Lakens, 2021). The first of these hurdles can be reduced by increasing the range, ease, and flexibility of procedures for power analysis. The second hurdle can be somewhat reduced with the use of sequential analytic designs (Wald, 1945). In sequential analytic designs, data is analyzed at multiple stages of the data collection process, and the researcher decides whether to continue data collection or not depending on the results. Such designs can (but do not always) allow a researcher to stop 
sampling after fewer observations relative to traditional fixed designs, with comparable power and false positive rates.

As noted, a range of tools are currently available for power analysis, and the increasing recognition of the value of sequential designs amongst psychologists has also spurred the publication of tutorials and guidelines for several sequential analytic procedures (e.g., Lakens, 2014; Schnuerch \& Erdfelder, 2020; Schönbrodt \& Stefan, 2018; Schönbrodt \& Wagenmakers, 2018, see www.sequentialtesting.com for a collection of articles). Current expositions of these areas predominantly focus on either frequentist or Bayesian null hypothesis testing procedures (i.e., using $p$-values or Bayes Factors as decision criteria). To our knowledge, few papers have considered power analysis from the perspective of Bayesian parameter estimation, with John Kruschke and colleagues being notable exceptions (Kruschke, 2013, 2014; Kruschke \& Liddell, 2018).

In parameter estimation approaches, also known as 'new statistics' (Cumming, 2014; Kruschke \& Liddell, 2018), the primary focus is not so much on making a binary decision about the presence or absence of an effect, but rather on generating estimates of parameters that might underpin observed data. For example, rather than simply saying an effect exists or not, one might provide a parameter estimate for a range of possible effect sizes consistent with the observed data. In Bayesian new statistics, this estimate takes the form of a posterior distribution of possible parameter values consistent with the underlying model's prior and the data with which that prior is updated (e.g., a posterior distribution of Cohen's $d$ ). This posterior distribution for the parameter estimate can be displayed directly to the reader, and also summarized in a variety of ways. For example, one might be interested in the $95 \%$ highest density region (HDI), which spans the $95 \%$ most probable values of the posterior. Alternatively, one might want to determine what percentage of the posterior lies above or below a particular value. 
If we are simply seeking to get a parameter estimate, then what decision criteria might we use to determine power? Two well-subscribed approaches are to seek to accept/reject a certain parameter value or range of values (Kruschke, 2018), or to achieve a parameter estimate with a desired level of precision (Kruschke, 2014; Maxwell et al., 2008). For example, in a comparison of two independent groups, we might anticipate an effect size of around $d=.55$, and feel that an effect lower than $d=.1$ is too small to be of interest. We might then conduct a power analysis to determine what sample size we would need, given the anticipated effect size or range of effect sizes, to achieve a 95\% HDI for the effect size that excludes $d<.1$. Alternatively, we might want to estimate the effect size to a particular degree of certainty, say a 95\% HDI for Cohen's $d$ spanning at most .5 standardized units, and wish to know what sample size would be required to achieve this.

While the emphasis in interpretation of results from Bayesian estimation may be upon the plausible sizes of the estimated effects, the decision to accept/reject a certain parameter value can be understood from a power analysis perspective as akin to a hypothesis test for the parameter of interest being above/below a certain threshold of interest, or outside/within a range of practical equivalence (ROPE). Hence, decisions based on these Bayesian estimates can be subject to false positives and negatives due to unexpected extreme values just like frequentist hypothesis tests, and these probabilities can be incorporated into power analyses (e.g., we conclude our study with an indication that the underlying effect size is greater than $d$ $=.1$, when it is not). Decisions based around the precision of an estimate are typically robust to the chance collection of unrepresentative samples, as these will not suddenly cause the decision criterion to be reached (Kruschke, 2014). Hence, some may argue that precision is the most valid target for power analyses.

Whatever one's perspective on this issue, the approach to power presented in this paper allows planning for precision or for the acceptance/rejection of parameter values, as well as for the control of error rates if the latter is chosen. The present approach expands upon 
previous work regarding power in Bayesian estimation in several ways: 1) We introduce the use of the $R$ package furrr (Vaughan et al., 2021) to leverage parallel processing capacities of computers to reduce simulation times; 2) Previous work has primarily urged researchers to generate valid hypothetical data sets, which are then analyzed, and the posterior distribution of the analysis is used to generate new hypothetical data sets for simulated power analyses (Kruschke \& Liddell, 2018). This approach is entirely consistent with what we propose and may also be used, but we demonstrate power analyses based upon simple and direct specification of different effect sizes, or a range of plausible effect sizes, which some may find more approachable. Our intention is not to advocate for any one approach or another but to show what is possible if desired; 3) We show how to calculate power given different design choices, e.g., power to detect an effect given a presumed underlying effect size or power to detect a smallest effect size of interest; 4) We consider how different types of false positive can be incorporated into a power analysis, and how decision criteria can be flexibly adapted to control such error rates without needing to perform new simulations; 5) We show how power estimates from fixed $N$ designs can be extended to estimate power for sequential designs, again with error control; 6) We show how to incorporate stopping based upon accepting a null value or value of practical equivalence. One final consideration is that we use the increasingly popular $R$ package brms (Bürkner, 2017). This is not an advance upon previous work, as direct programming in Stan or JAGS ultimately allows greater flexibility of model construction. However, it may make such analyses more approachable - for example, brms allows familiar model specification analogous to the commonly-used package lme4 (Bates et al., 2007).

In the sections that follow, we cover the design of a basic fixed $N$ power analysis with a presumed underlying effect, geared towards accepting/rejecting a particular effect size. We will then show how to extend this power analysis to calculate power in a group sequential design for the same research question. We briefly show how such a sequential design could 
lead to efficiency gains relative to typical fixed $N$ designs. We then show how one might instead calculate power for detecting a smallest effect size of interest, or how one might assess sensitivity to detect a range of different effect sizes. For all these examples, we show how the exact same data can be used to determine power given any decision criterion based on the posterior distribution of interest, without needing to undertake more simulations (provided one asks for that posterior parameter estimate to be returned in the original simulations). We then consider the presented approach's limitations and how it might be extended. Finally, we briefly discuss how sequential analyses also involve aspects beyond the merely statistical/power-related, which should also be considered in experimental design.

\section{Generate data for power simulations}

As noted, there are several ways that could be used to generate data for power analyses. In the following example, we will use the idea of generating data from a distribution reflecting an anticipated range of effect sizes - specifically a distribution of Cohen's $d$ for independent samples with a mean of .55 and a standard deviation (SD) of .125. Hence, the effect size of .55 is typical, $95 \%$ of our effect sizes are anticipated between .3 and .8 , and almost all come between .175 and .925 . This distribution might be a posterior distribution of effect sizes based upon previous research. Alternatively, it might be an educated and conservative estimate based upon knowledge of effect sizes in the research area of interest, or drawing upon a solid underlying theory. Another way of generating data is to create a set of observations that matches one's expectations, run a Bayesian analysis of that data, and then generate new data based upon the posterior distribution of that analysis (Kruschke \& Liddell, 2018). Finally, one might pick several different effect sizes of interest and generate data based upon those.

There is considerable debate about how one should select effect sizes for power analyses. For example, simply picking a single effect size based on previous research might lead to inflated expectations of effects due to publication bias and other factors (Anderson et 
al., 2017). The aim of this tutorial is not to favor any particular means of deciding on estimated effects for data generation - any approach that can be used to generate a large number of simulated data sets can utilize the overall power method presented here. We will focus on the how, and leave the 'should' to more specialized treatments of that specific issue.

First, we load up the tidyverse $R$ package for our basic data wrangling and visualization (Wickham et al., 2019). We use the rnorm() function to generate 1500 data points from a normal distribution with mean of .55 and SD of .125 and put them in a tibble (a type of dataframe used in the tidyverse that has some nice features - when you read 'tibble' below, you can just think 'dataframe') that also pairs them with a simulation number:

effect.sizes <- tibble (effect.size = rnorm(n= 1500, mean =.55, sd = .125), $\operatorname{sim}=1: 1500)$

We can visualize the distribution of effect sizes as follows:

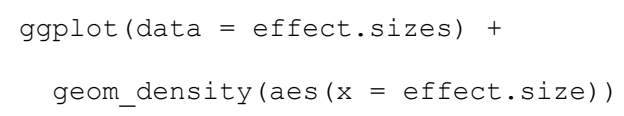

The custom data.maker() function is designed to take a value of Cohen's $d$ given to it and generate data of a chosen max sample size per group for two independent groups: a reference group and a group that differs from it by the given effect size. We can specify a mean and standard deviation for the reference group from which the second group will deviate. In this case, to more easily work with standardized effect sizes we will simply give the reference group a mean of 0 and SD of 1 . The second group will be generated with a mean from our distribution of expected effect sizes and the same SD. A map function ${ }^{1}$ is used to

\footnotetext{
${ }^{1}$ Map functions and other members of the purrr family are extremely useful and powerful; an introduction to their usage can be found at https://rstudio.cloud/learn/primers/5. furrr features similar functions that run in parallel.
} 
apply data.maker() to every row of our effect.sizes tibble, returning a new tibble with all of our simulated data:

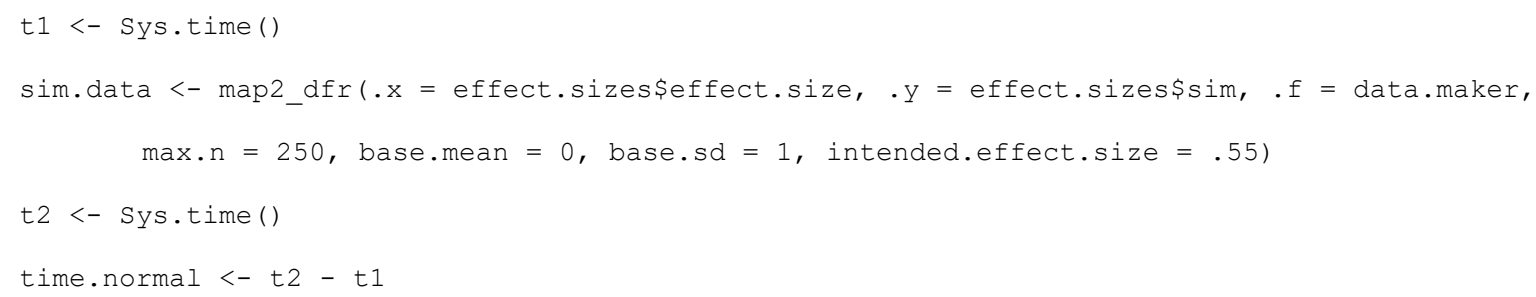

In this case, we have timed the process, which takes about 11.5 seconds on my computer (stored in 'time.normal'). When we start running regressions on this data, every second will count, and so it is worth already introducing the furrr package (Vaughan et al., 2021) to leverage the parallel processing capabilities of most computers. We can run the same map function in parallel on most laptops/computers to save considerable time, and it takes very little extra code. We load the furrr package, tell it to run in parallel with the 'multisession' command, and provide a seed to furrr_options(). We then use the future_map2_dfr() function and add in our seed options, as opposed to simply map2_dfr():

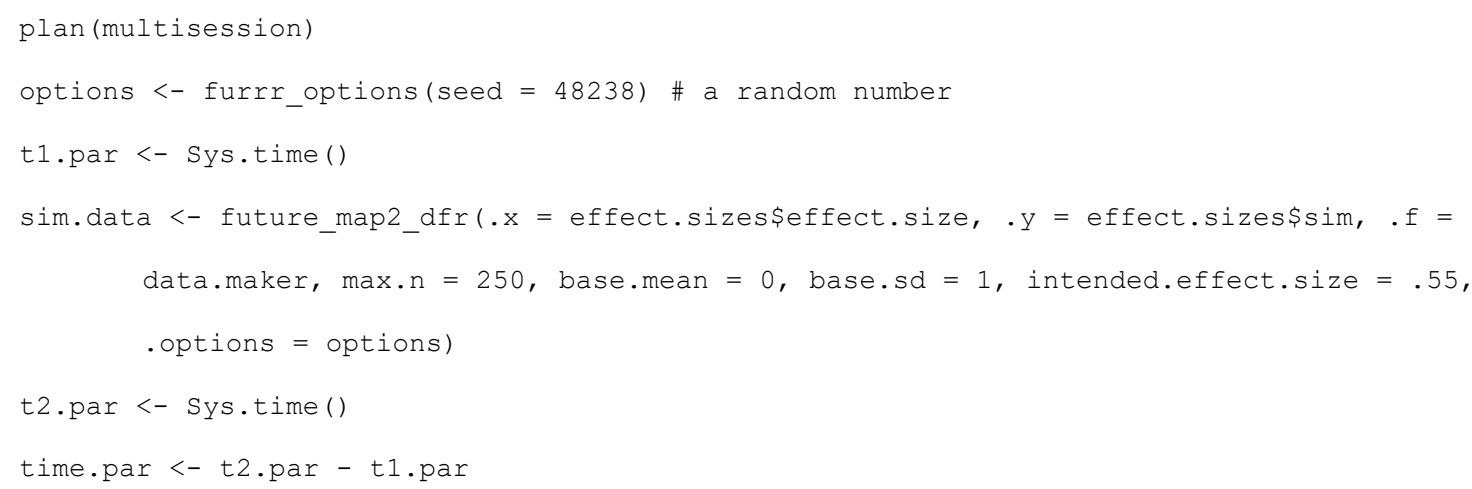

Looking at the value for time.par shows this has taken just 2.8 seconds (on my computer). This is a great efficiency gain, and can prove tremendously valuable when running a large number of regressions later, in which the difference might be more like 20 minutes vs. 
3 hours. We are also going to increase the memory limit in $R$ to ensure that our later regressions do not exceed capacity:

memory.limit (80000)

To consider a possible false positive scenario, we generate a further reference data set, made with a distribution of effect sizes from a normal distribution (mean $=0$ and $\mathrm{SD}=.033$ ), capped to be at or above 0 and less than .1 (i.e., from 0 to right below what the researcher has deemed a minimally important effect size for their purposes). Again, other means of choosing null or negligible effect sizes could be considered, such as a point null at 0 , a posterior from previous research or theory, or constructing data one would consider to be essentially a 'null' effect and running a regression on this to recover the underlying parameters, and generating data sets from that. We generate these new sets of data and combine them all with our previous simulated data set, and finally group and split the tibble:

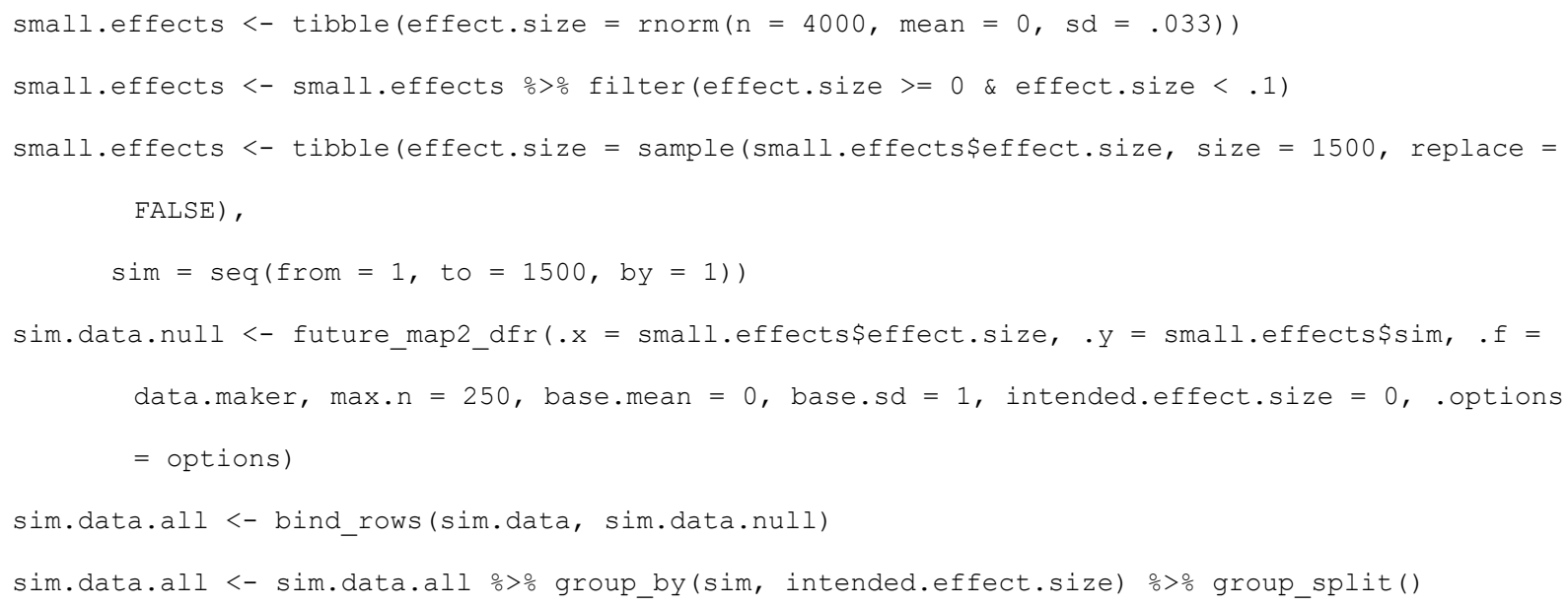

The object 'sim.data.all' is now a list of 3000 data sets, each corresponding to one row of our requested effect sizes. We are ready to start analyzing these data sets. 


\section{Setting up our base regression model}

We will use brms (Bürkner, 2017) to analyze the data. This requires also setting up one's computer to run Stan (more specifically, RStan), which will do the legwork of the Bayesian analyses. To install RStan, follow the instructions at https://github.com/standev/rstan/wiki/RStan-Getting-Started ${ }^{2}$. Next, we set up the regression model that will be run over all of our simulated data sets. We will provide the model with another simulated data set to confirm that it runs appropriately:

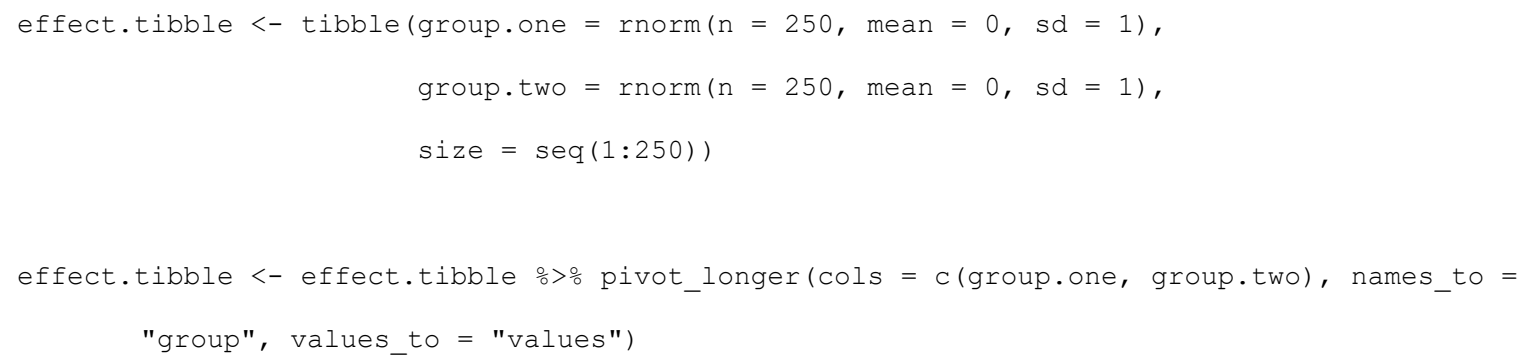

Our regression formula is very simple, requesting the prediction of 'values' (each simulated response) from the intercept (1) and group designation (group):

reg.form $<-$ values $\sim 1+$ group

As this is a Bayesian model, we can also see what priors can be set for it using the get_prior() function:

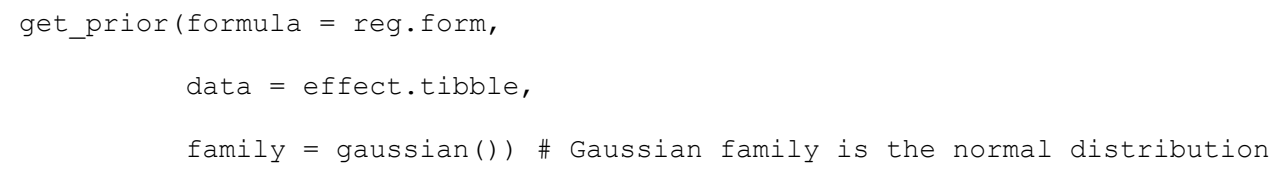

\footnotetext{
${ }^{2}$ If you do not want to or cannot install RStan, the OSF files include the regression output so that you do not have to run it yourself. Output from the regressions on all the simulated data sets are also included. $\underline{\text { https://osf.io/32uxa/?view only }=\mathrm{d} 8930640 \mathrm{e} 62 \mathrm{e} 492 \mathrm{~b} 96 \mathrm{~d} 5 \mathrm{~d} 2 \mathrm{da} 9 \mathrm{eb} 8839 \mathrm{c}}$
} 


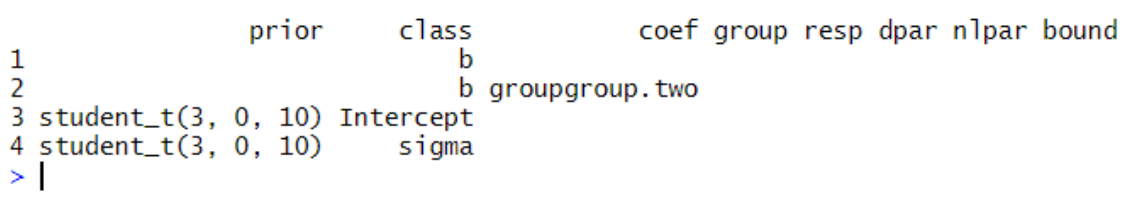

Figure 1. Output in the R console showing what priors can be set for the regression model

The output in the console (Figure 1) shows that we can set priors on the intercept ('Intercept' in the console, which refers to the group.one mean), the deflection from the intercept for group.two ('groupgroup.two' in the console), and the standard deviation around the means ('sigma'). The default priors for these parameters are extremely broad ('student_t $\left.(3,0,10)^{\prime}\right)$, and so we will constrain them more with weakly informative priors:

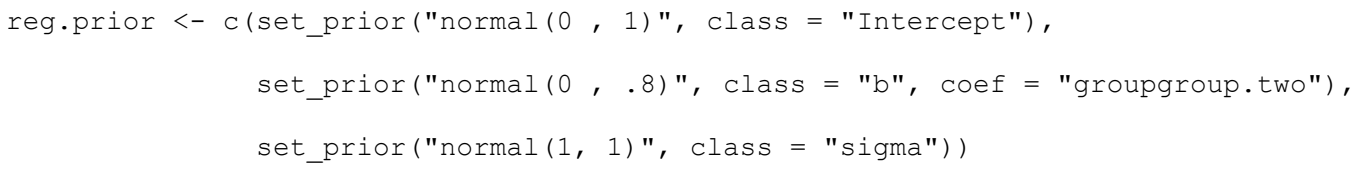

These priors simply restrict the prior parameter search space to less extreme values than the defaults. For example, the prior on the intercept means that $95 \%$ of prior probability of values for the mean of group one will be between -2 and 2 , and $95 \%$ of the prior probability for the group two difference will be from -1.6 and 1.6. These are not excessively conservative priors but do rein in some of the extreme differences between groups that would be entertained by the default options. The priors are not fully informative because we have not, for example, specified that we anticipate that group two is greater than group one: our priors are agnostic as to a possible difference between groups, even though we might expect there to be such a difference. This is important for convincing a skeptical audience. Other more or less informative priors might be considered depending on one's goals and prior knowledge, or to assess the sensitivity of one's conclusions to reasonable variation in the 
priors. For the purposes of demonstration, however, we will move forward with these. We now run a brms regression on this single data set:

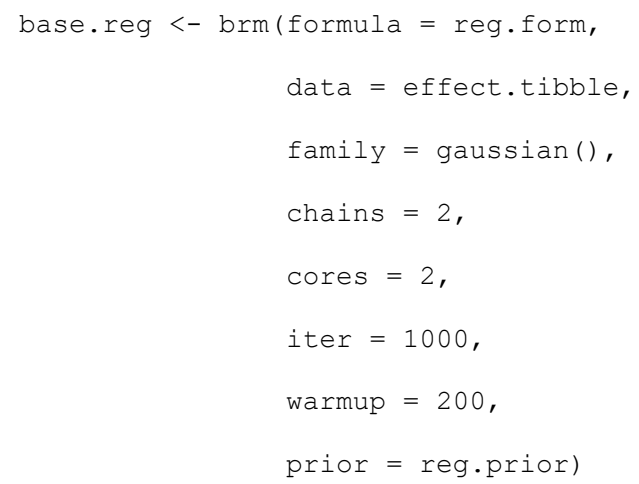

A quick look at the model output in the console (Figure 2) shows that our regression has worked nicely, recovering the underlying intercept and effect of group (both in this case around 0) and standard deviation (around 1). If we wish to increase the effective sample size, we can request more iterations. If, when running our larger number of regressions below, we want to just check that the code is working and get a quick look at some basic results, we can reduce the iterations to make the regressions run more quickly, but with less robust results. We have a decent effective bulk sample size for each parameter, and the chains have converged with an Rhat of around 1.00 (Rhat should not exceed 1.05 and can typically be made close to 1.00$)$.

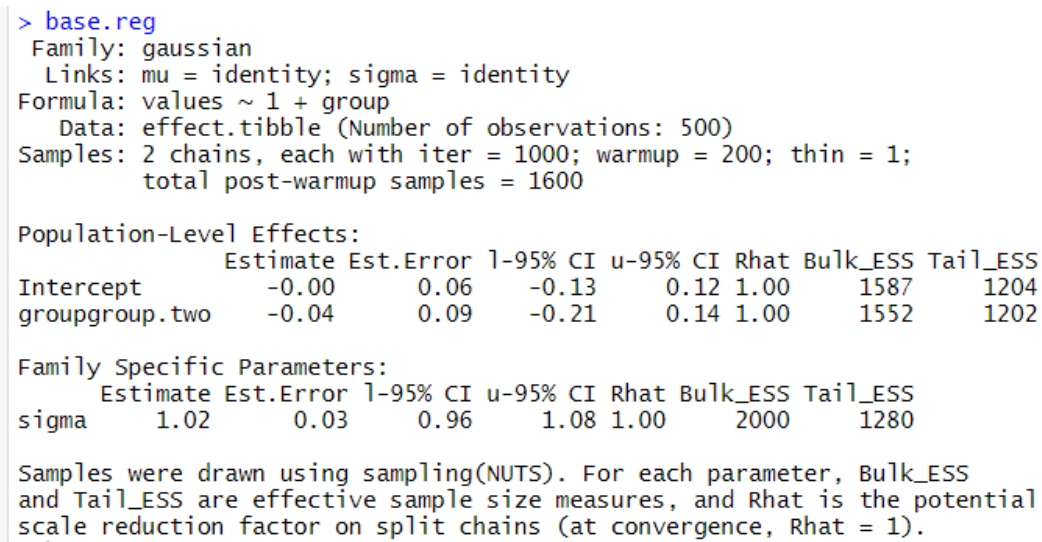

Figure 2. Output in the R console showing the basic results of the regression model. 


\section{Running the regression model on many simulated data sets}

We now turn to the most time-consuming part of the power procedure: running the regression over the 3000 data sets. The reg.maker() function is used for this purpose. The function takes a simulated data set, and the base regression model we generated above. If you look inside the function, you can see that we are asking that for each data set, we run the regression for the first 50, then 100,150, 200, and 250 participants per group. This is done so that we can observe the results given those respective sample sizes, to get an estimate of the power achieved with each size. The second thing to note is that we generate a posterior distribution for the effect size within the function: for each regression that is run, we take the posterior distribution for the effect of group and divide it by the overall standard deviation, to generate a posterior distribution of Cohen's $d$. Generating this posterior will be crucial for our purposes as it is what we are basing our inferences upon, but in principle one could request a number of other parameters. In more complex regression models, for example, one might extract the estimate for the effect of some continuous predictor variable on the outcome. The final thing to note is that we use the update() function of brms to 'update' the base regression model with each new data set. Contrary to what one might expect given the name, this 'update' does not add each data set in alongside the previous data set or combine them in any way. The base regression model is run from scratch again each time using the newly provided data, but importantly it does not need to compile the model in $\mathrm{C}++$ each time it runs, which shaves a huge amount of time off of the process. We use the future_map_dfr() function to pass each of our 3000 data sets to the function and run the analyses in parallel:

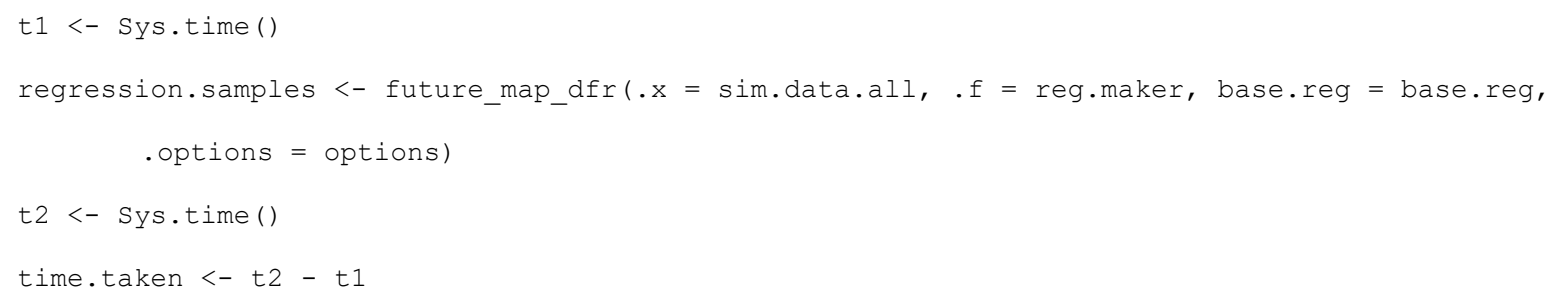


On my computer, time.taken shows that this took about 11 minutes - impressive given that we have passed 3000 data sets to the function, and each is analyzed in 5 regressions $=$ 15000 Bayesian regressions in 11 minutes. Your computer may run differently, and so it can be worth testing out a lower number of simulated data sets at first to get a sense of how long it is likely to take. The regression.samples object is now a massive tibble containing all the posterior distributions for the regressions, with columns indicating the corresponding true effect sizes and parameter estimates for the intercept, group effect, SD, and crucially the estimate of Cohen's $d$.

\section{Flexibly considering power and decision criteria}

Because we have whole posterior distributions at hand, we can consider some different inference goals, and observe the probability we have of achieving these goals given different sample sizes (i.e., the power). To do this we must summarize the posterior distributions and request some informative metrics. To get HDIs, we use the hdi() function from tidybayes (Kay, 2020). To summarize each analysis, we group the tibble by the intended effect sizes (around .55 or between $0-0.1$ ), sample sizes, and simulation, and use the summarise() function from the tidyverse to summarize each run of the regression:

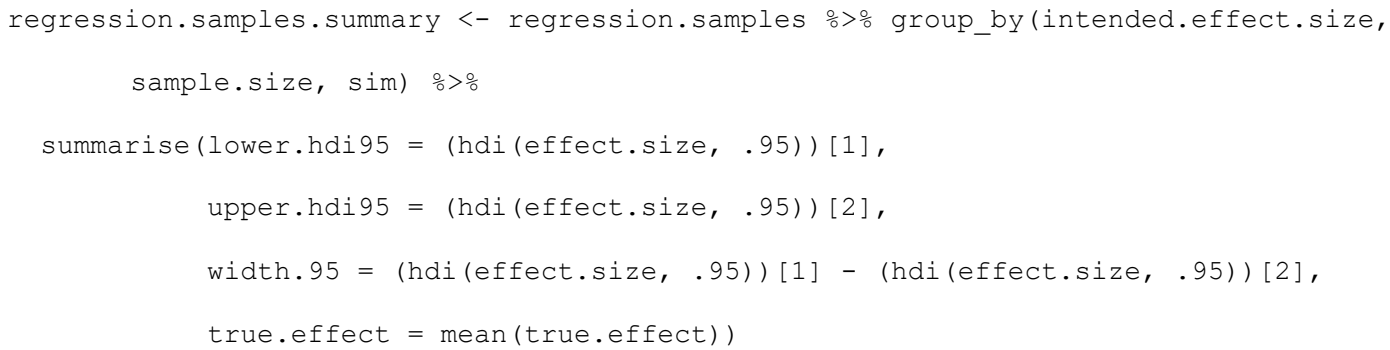

To determine how likely we are to achieve an inference goal, we can select a goal and calculate the proportion of times across simulations that we would have met it if we had ended the experiment at different sample sizes - e.g., how often do we have a 95\% HDI whose lower bound is equal to or greater than .1? 


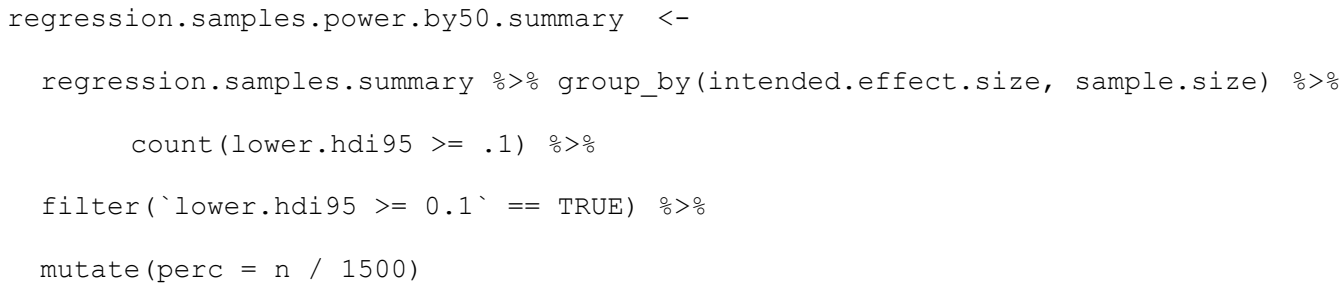

The column 'perc' now shows this proportion. For the effect sizes around .55, this reflects our power to correctly detect that Cohen's $d$ was greater than 0.1 . For the effect sizes between $0-0.1$, this proportion would be a false positive rate or rate of misleading evidence: we might in these cases conclude that the effect size was greater than .1 when in fact it was not. We can also consider how often we have 'accepted' an estimated effect size of less than .1 with this same approach (naming a different object, changing $>=.1$ to $<.1$, and using the upper bound of the 95\% HDI in the code above - code removed for space but included in the supplement). In the case of a true effect around .55, this would be a false negative conclusion, and for the effect sizes below .1 would represent an accurate conclusion of the effect size being less than .1 .

Plotting these summaries shows us the proportion of times we have achieved the goal of rejecting an effect of less than $d=.1$, given the different underlying true effect sizes, as well as how often we would conclude that the effect is probably less than .1:

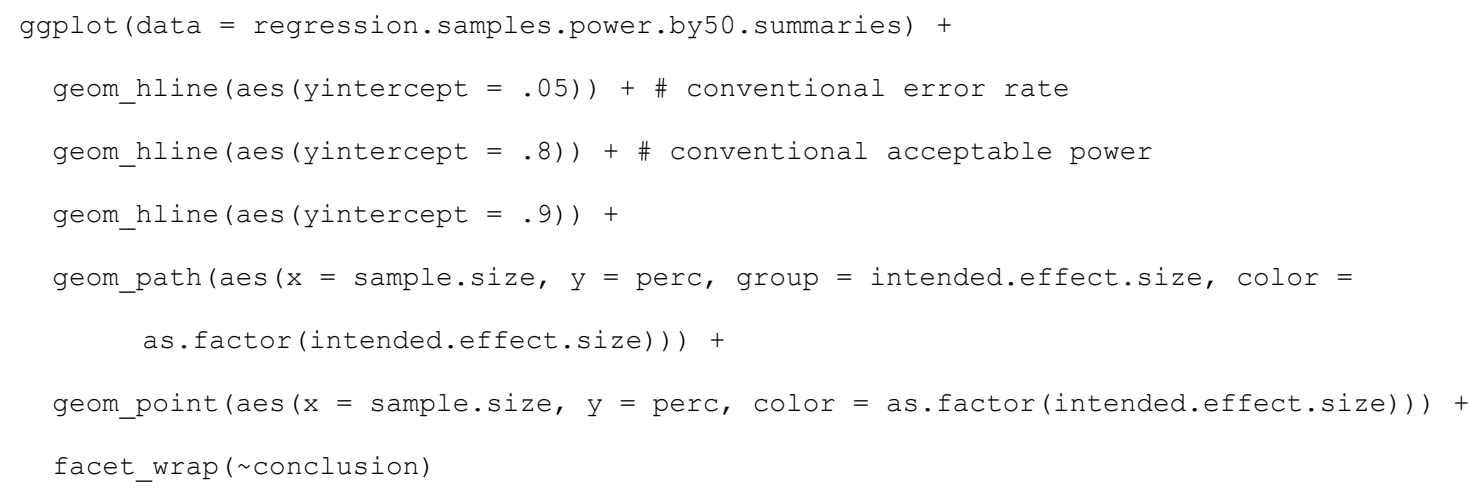




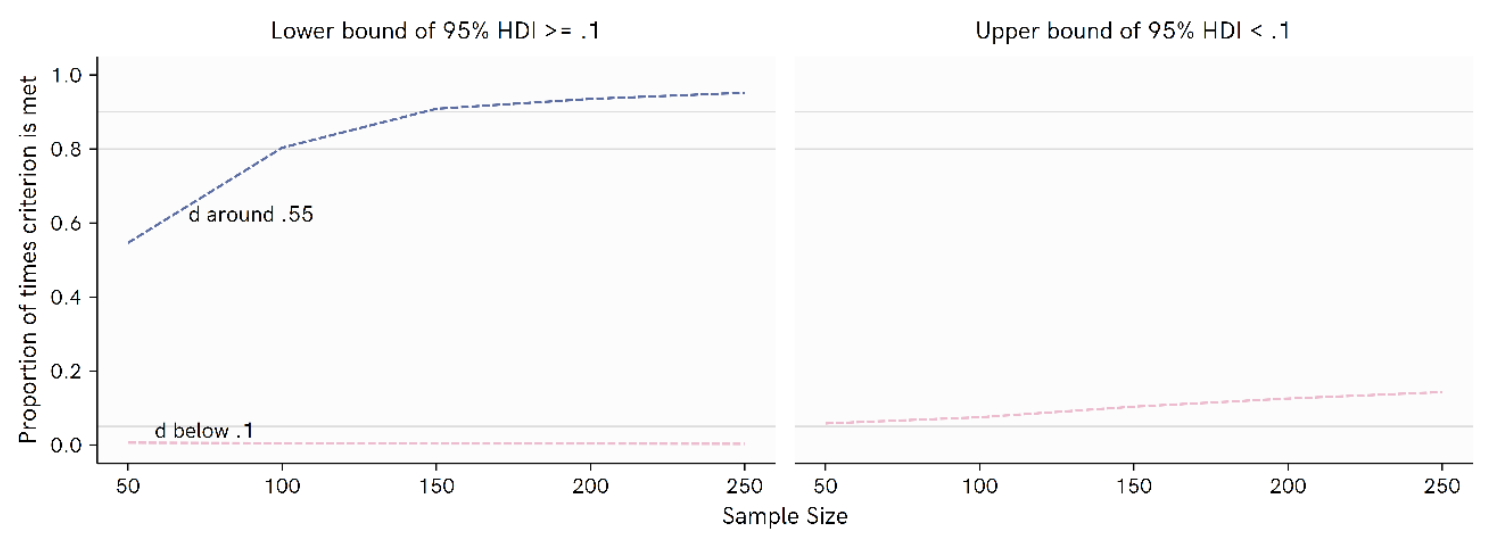

Figure 3. Assessment of the proportion of times across simulations that decision criteria are met with different true underlying effect sizes, from 50-250 participants per group in batches of 50 per group. The horizontal lines reflect conventionally accepted rates of misleading evidence/‘false positives' (.05) and power (.80 or .90$)$.

From Figure 3, we can see that with sample sizes of 100 and 150 per group, and the true effect size matching our distribution around .55, we would achieve the goal of the 95\% HDI excluding .1 about 80 and $90 \%$ of the time respectively. The false positive rate for effect sizes between 0 and .1 are very low at all sample sizes. We can also see that we would almost never have a false negative effect of assuming an effect lower than .1 when the true effect comes from the distribution around .55 , and could correctly accept the effect size as being lower than .1 about $13 \%$ of the time if the effect size is actually from the distribution close to 0.

If our goal were instead to achieve an estimate of the effect size that is less than .6, .5, or .4 standard deviation units, we can see that we would need sample sizes of around 100, 150, and 250 per group to achieve this, given a true effect size of around .55: 

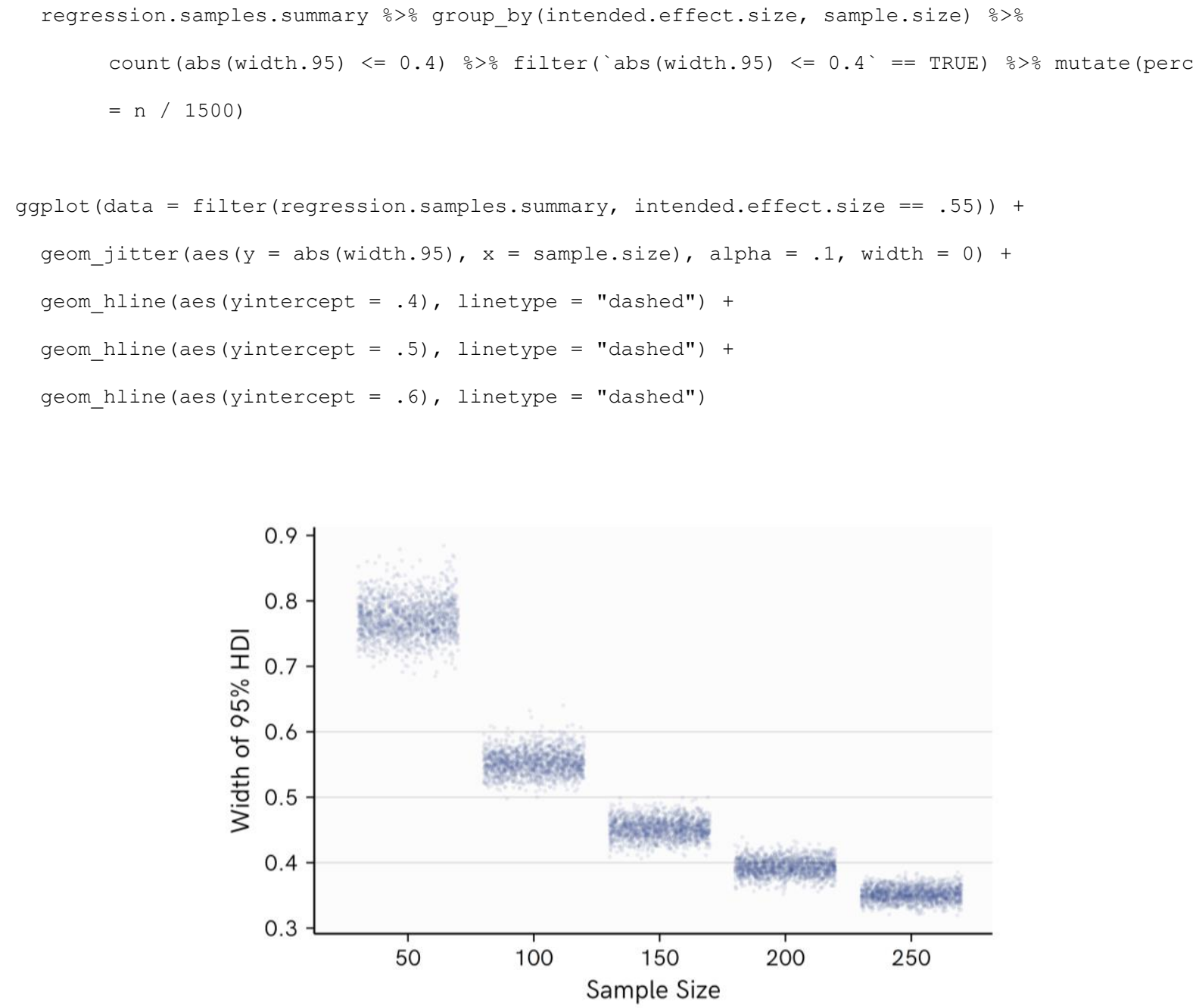

Figure 4. Width of 95\% HDIs for Cohen's $d$ across samples for different sample sizes, and an underlying effect distributed around .55. Greater sample sizes result in narrower HDIs.

The output from the summary tibbles above show that we tend to go quite suddenly over a sample size threshold at which we become overwhelmingly likely to achieve our goal, with almost no chance at the previous sample size. This is graphically displayed in Figure 4 .

Before continuing to assess the likelihood of achieving our inferential goals in sequential designs, we might think of whether we wish to get a more fine-grained assessment of power. Given that we already have 90\% power to achieve our goal of excluding $d<.1$ from the $95 \%$ HDIs at 150 participants per group, we may not need simulations of samples sizes of 200 and 250, and we might also want to make assessments with some smaller increments in 
the sample size. We will use the reg.maker() function again for this, and pass it an argument for the sample size break points with a lower maximum and smaller increments in each sample size increase. Running this analysis took about 34 minutes on my computer. When running your own power calculations, it can be an idea to first run smaller numbers of regressions with a large range of sample sizes, to help pinpoint a reduced range of sample sizes within which one could make a more fine-grained search (it would be annoying and time consuming to run a very large number of regressions in increments of 10 participants from 10300 to discover that one need only have gone up to a sample size of 100 , or that one would probably need to go up to 350$)$.
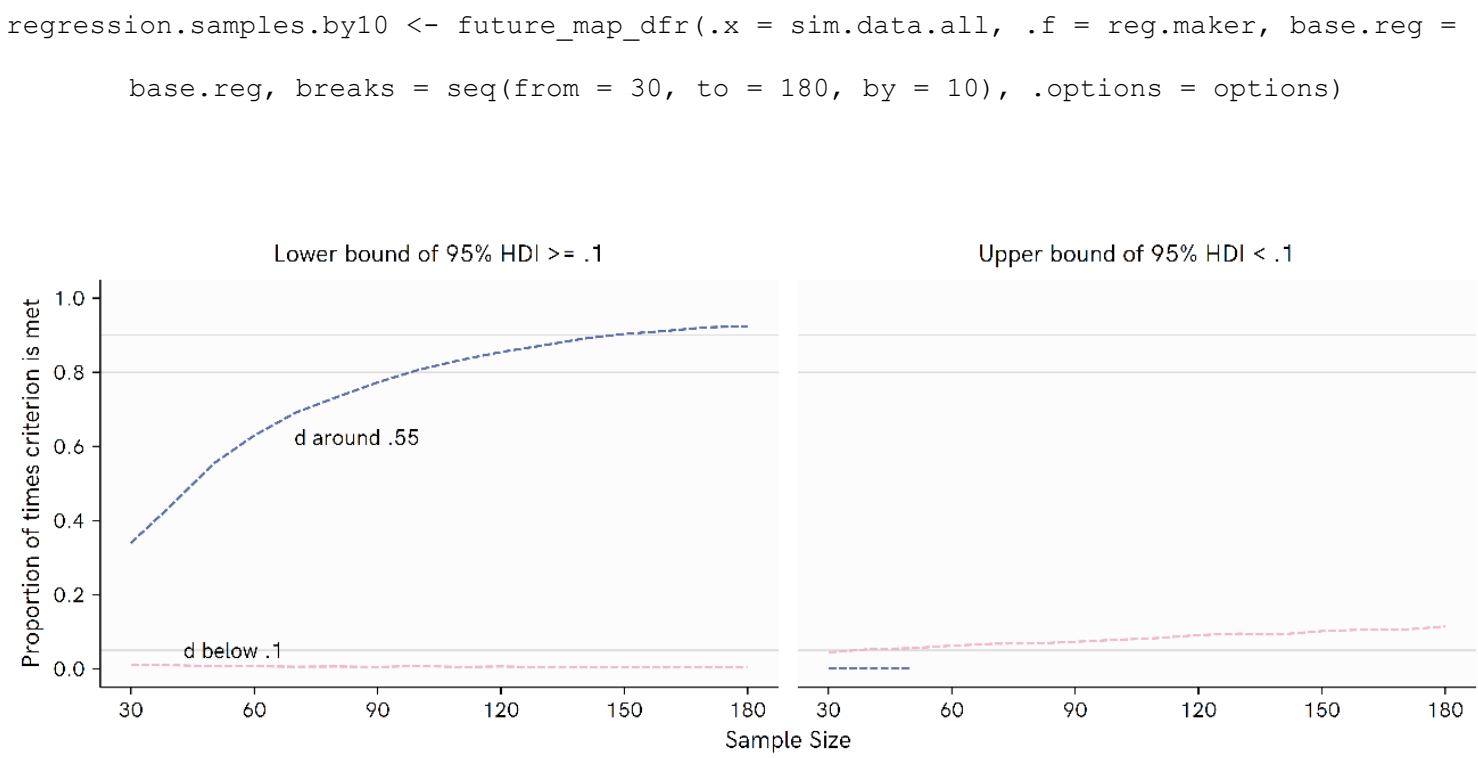

Figure 5. Assessment of the proportion of times across simulations that decision criteria are met with different true underlying effect sizes, from 30-180 participants per group in batches of 10 per group. The horizontal lines reflect conventionally accepted rates of misleading evidence/‘false positives' (.05) and power (.80 or .90).

Figure 5 shows our power and rates of misleading evidence for this new set of sample sizes. To estimate power if we were to analyze the data sequentially, we can use the sequential.power() function. This function takes the power summaries from above (split by 
the intended effect size and simulation), an indicator of what metric is being assessed (e.g., the lower bound of the 95\% HDI), a threshold this value is being compared against (.1) and logical operator for the comparison (">="), and finally a vector indicating at what break points the data is to be checked at:

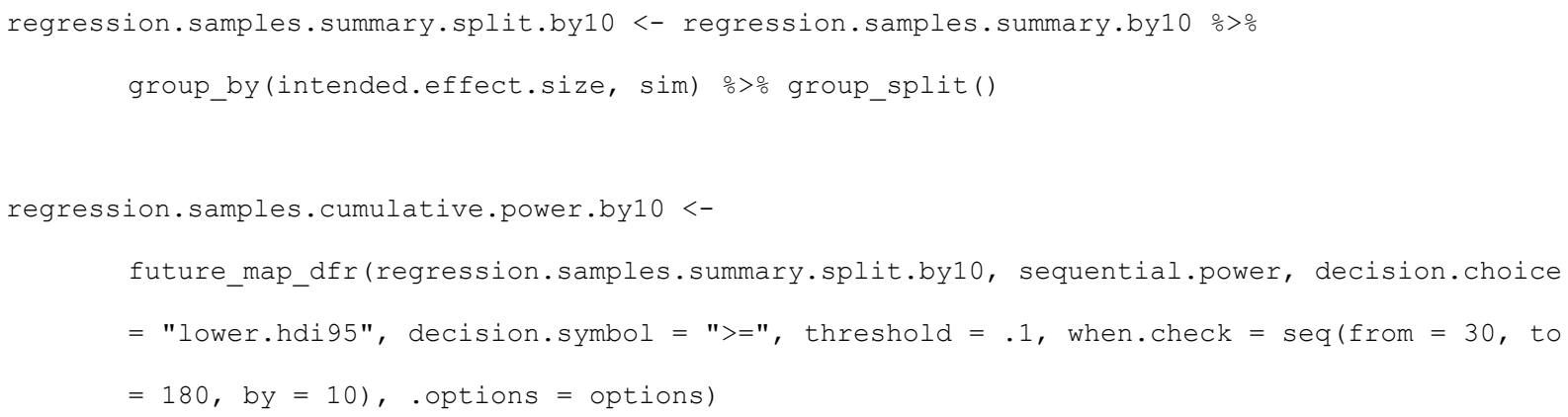

Code presented in the code supplement then does the same but assessing for the conclusion that the upper 95\% HDI is below.1. We can then plot this sequential power alongside that fixed $\mathrm{N}$ power for comparison:

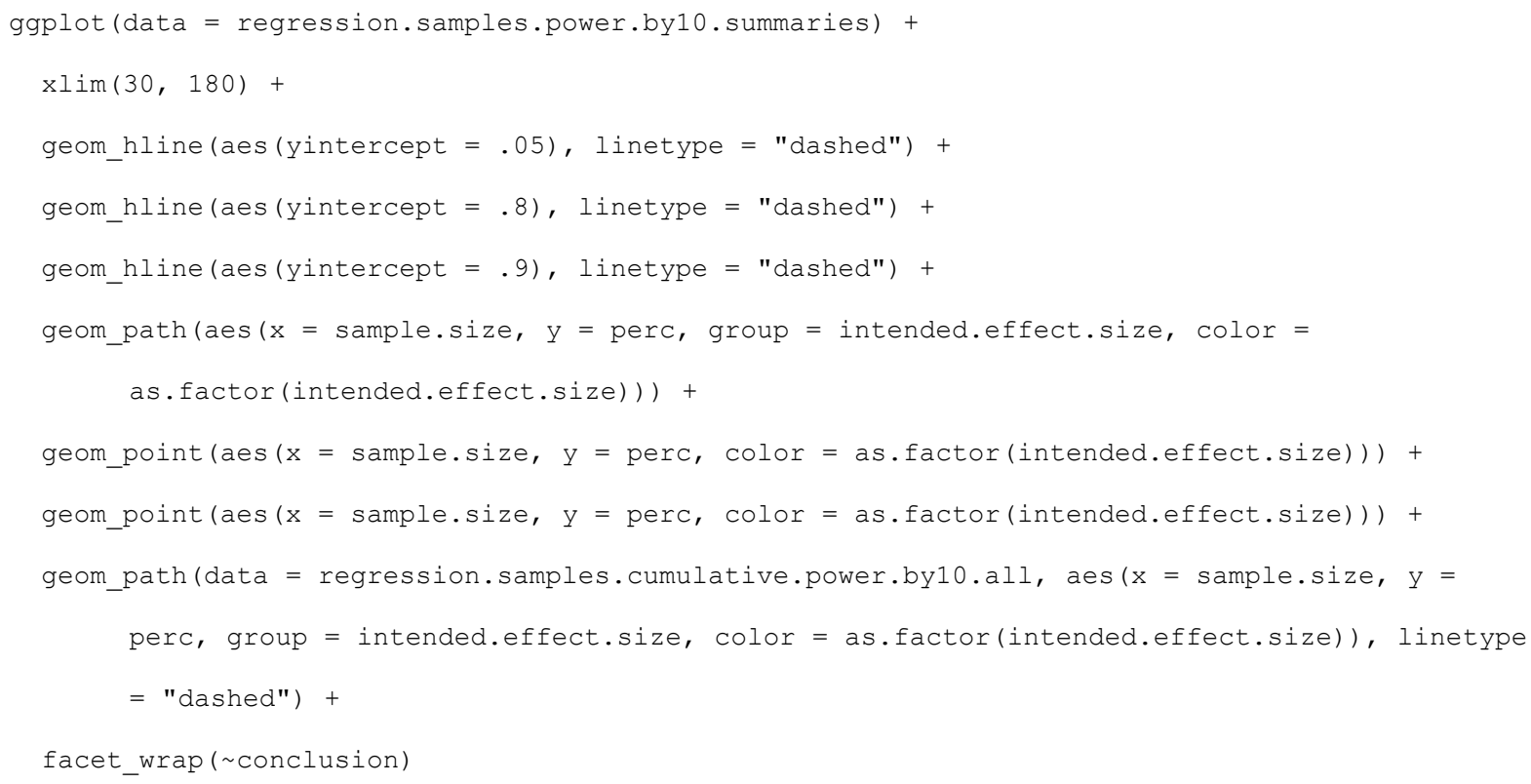




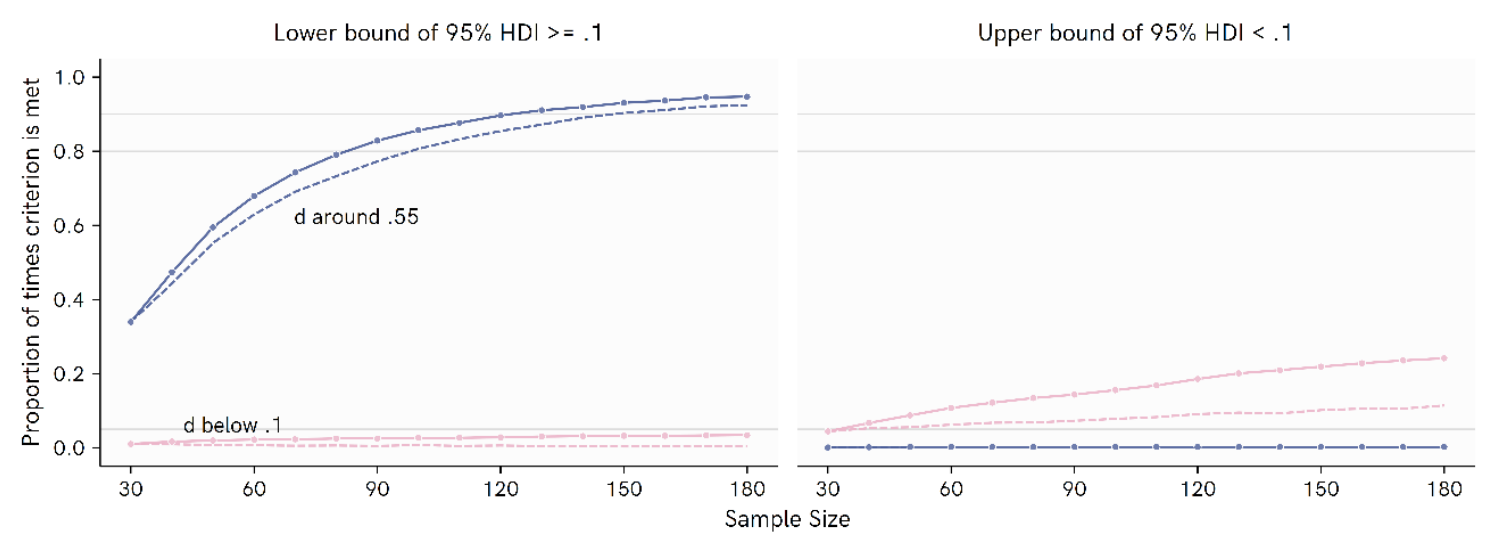

Figure 6. Assessment of the proportion of times across simulations that decision criteria are met with different true underlying effect sizes, from 30-180 participants per group in batches of 10 per group. Dashed line depicts the fixed $\mathrm{N}$ assessment, whereas the solid line with points depicts sequential testing at each sample size. The horizontal lines reflect conventionally accepted rates of misleading evidence/'false positives' (.05) and power (.80 or $.90)$.

From comparing the solid line (the probabilities for reaching decision thresholds in a sequential analysis) vs. the dashed line (the probabilities of having reached a decision if setting a fixed stop at that sample size) we can see that the sequential procedure has greater power to reach a decision criterion (Figure 6). There is also an increase in the rates of misleading evidence, though in this case the rates still remain below $5 \%$, owing to the relatively stringent decision criteria (see the next section for an example of inflated misleading evidence with less stringent thresholds). Perhaps more importantly than the moderate increases in power is the opportunity the sequential design gives us to stop data collection having used fewer resources. To consider the case in which the true effect size matches our distribution around .55 , with a fixed maximum sample size of, say, 120 per group (over $80 \%$ power), we of course have a guaranteed sample size requirement of 240 
participants total. In a sequential design, we could well end up going to this chosen maximum, but with the option of stopping every 10 participants per group, our mean sample size requirement is 117.5 total (rounding to get a batch of 10 per group, this might end up at 120 participants, 60 per group). If the effect size were around 0 , then there would also be the opportunity to cease data collection early when the effect size is estimated to be below .1. Even without any direct increase in power, this possibility of curtailing sampling early makes a sequential approach very appealing.

\section{Power for a smallest effect size of interest and sensitivity to multiple effect sizes}

In the example above, we imagined a researcher with some knowledge or expectation of a particular effect size, and simulated data based upon this. But what if we want to specifically design a study so that we can reliably detect the smallest effect size we care about? Or, what if we want to assess the sensitivity of possible designs to several different effect sizes? These options are also possible using the general approach laid out above. In this case, we can simulate data for several different effect sizes: $d=0,0.1,0.2$, and 0.3 . All code for this is provided with the code supplement, but not placed here as it essentially reiterates what we have done above. We use the data.maker() function to generate new data sets reflecting the different effect sizes. Then we use the reg.maker() function and run analyses in parallel over all the data sets. As we are looking at small effects and we do not already know what sort of sample sizes would be needed, we request the analyses to be run from 50 to 750 participants per group with breaks of 50. This is quite a time-consuming process: on my computer it took about 64 minutes, totaling $4 * 1500 * 15=42000$ Bayesian regressions. We could of course begin with fewer stopping points and fewer data sets to get a sense of the sample sizes we wish to hone in on in a more thorough analysis. We again summarize the posterior distributions with whatever criteria we wish, and run the sequential.power() function over the summaries to determine how often we achieve our desired stopping goal across the different effect sizes and sample sizes. To further demonstrate the general flexibility of this 
approach, and also some of the pitfalls of sequential analyses, we have chosen a different stopping criterion to those used above. Somewhat like a one-sided $t$-test, our stopping criterion in this example might be that the top-most $95 \%$ of the posterior distribution for Cohen's $d$ is greater than 0 (and the converse for a negative stopping rule - that the bottom $95 \%$ of the posterior is less than or equal to 0). Plotting this, we get Figure 7.

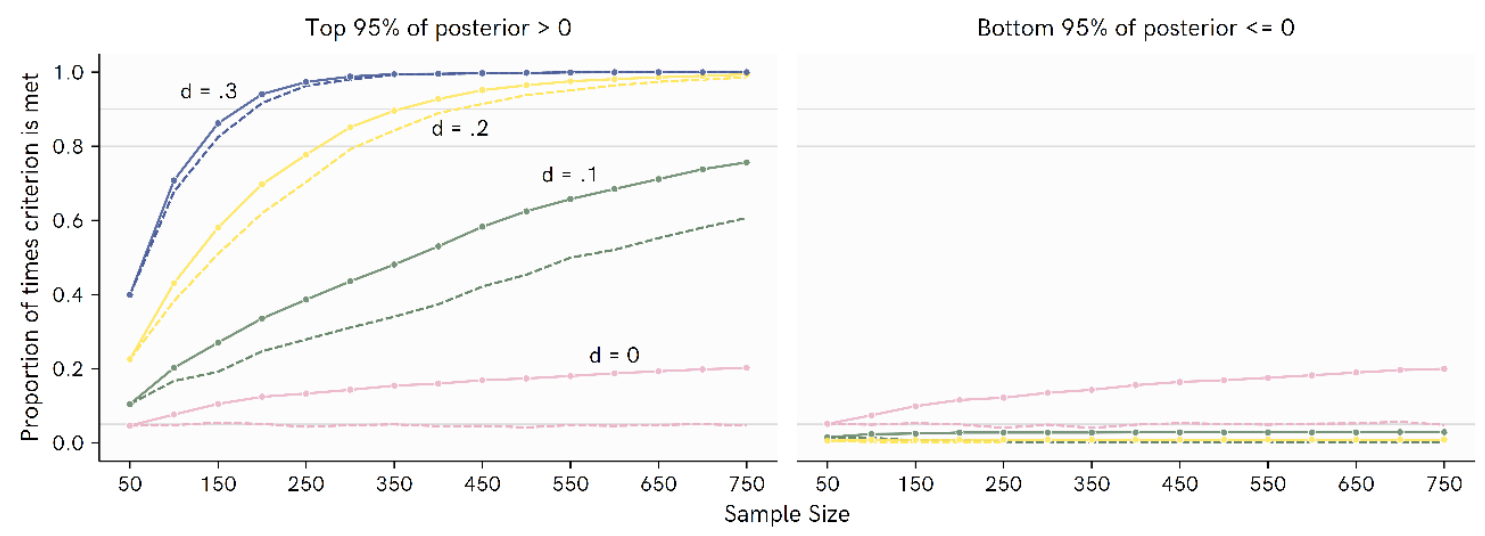

Figure 7. Assessment of the proportion of times across simulations that decision criteria (based on the top or bottom $95 \%$ of the posterior distributions) are met in a sensitivity analysis, from 50-750 participants per group in batches of 50 per group. Dashed line depicts the fixed $\mathrm{N}$ assessment, whereas the solid line with points depicts sequential testing at each sample size. The horizontal lines reflect conventionally accepted rates of misleading evidence/'false positives' (.05) and power (.80 or .90).

We can note several things from this graph. Firstly, our power to detect effect sizes of .2 or .3 is reasonable at samples less than $250-300$ per group, whereas in a fixed $\mathrm{N}$ design, an effect size of .1 would only be detected about $60 \%$ of the time even with samples of 750 per group. Secondly, we see that the sequential design again increases the likelihood that we can detect each effect size. Thirdly and importantly, we also see how the chosen decision criterion, in combination with a sequential design, quite dramatically inflates our rate of misleading evidence. The upper $95 \%$ of the posterior distribution for an effect size of 0 
triggers termination of the study on the presumption that effect sizes are likely above 0 up to $20 \%$ of the time. We might appreciate the increase in power that we achieve with sequential analysis, but we also do not want it to lead us astray. See the Misleading Evidence in Sequential Analyses section for a broader discussion of misleading evidence when performing sequential analyses. There are several ways we can rein in this false positive rate.

One approach is to use fewer checks of the data, such that there are fewer opportunities for misleading termination across the study. With the approach we have taken, this does not require re-running the regressions, only selecting different break points as an option to give the sequential.power() function. In this instance, starting checking at 150 participants per group and checking every further 150 results in a final false positive rate around $12.5 \%$ at 750 participants per group, relative to almost $20 \%$ when starting at 50 per group and checking every 50. However, given that our false positive rate using the current decision criterion is already at $5 \%$ in fixed analyses, we don't have much room to play with before the rate of misleading evidence gets to a level that is conventionally considered unacceptable. We can instead choose a stricter criterion, which will reduce both the chance that we detect legitimate effects and the rate of misleading evidence. Figure 8 shows the proportion of times we reach a decision when our criterion is that the upper $99 \%$ of the posterior is greater than 0 . 


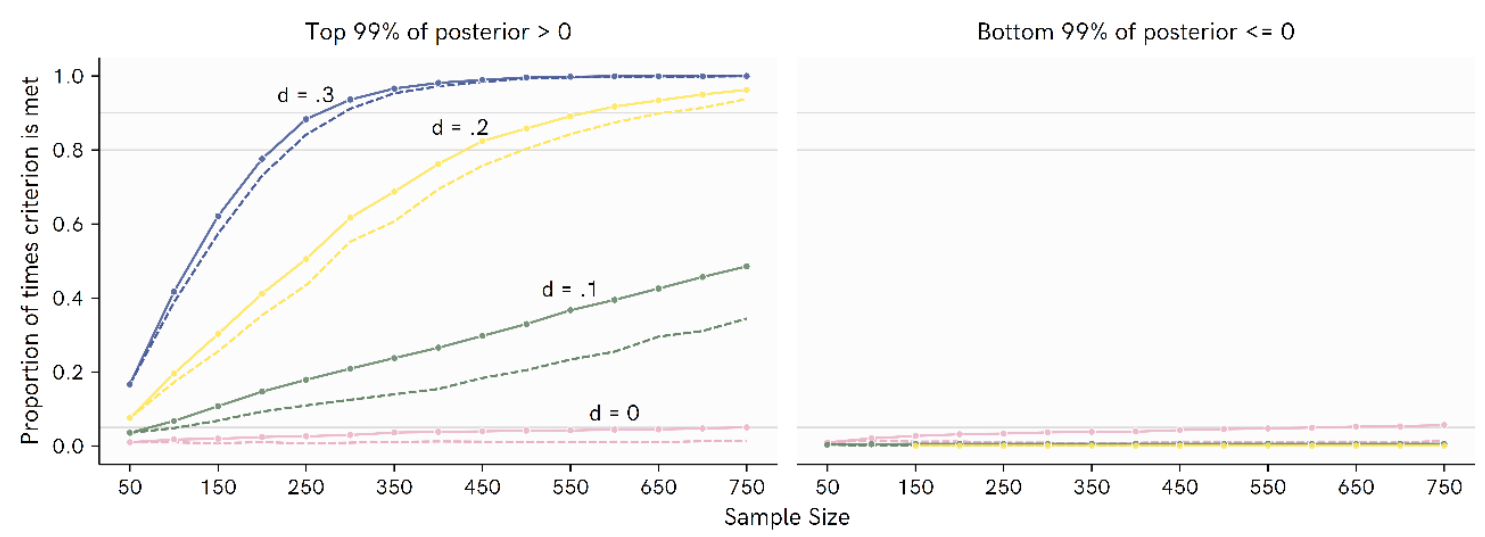

Figure 8. Assessment of the proportion of times across simulations that decision criteria (based on the top or bottom $99 \%$ of the posterior distributions) are met in a sensitivity analysis, from 50-750 participants per group in batches of 50 per group. Dashed line depicts the fixed $\mathrm{N}$ assessment, whereas the solid line with points depicts sequential testing at each sample size. The horizontal lines reflect conventionally accepted rates of misleading evidence/'false positives' (.05) and power (.80 or .90$)$.

With this more stringent decision threshold, we can dramatically reduce the rate of misleading evidence, with the false positive rate topping out at $5 \%$ for 750 participants per group. The trade-off for this is that we also reduce our power to detect true effects. Of course, if our smallest effect size of interest were .3 , we would probably consider a maximum sample size that is substantially lower than 750 , and could also thus select a slightly more liberal decision criterion as there would be fewer opportunities for misleading outcomes to accumulate. In addition, one could consider setting a different decision threshold to increase the prospect of terminating the study early if the results do not look at all promising. For example, one might be more liberal in accepting that the effect size is less than 0 or some other chosen small/null threshold. One must simply balance the chances of concluding that there is little meaningful effect with the potential for false negatives. The false negative rate is reflected in the proportion of times the negative threshold is reached when the true simulated 
effect size is above 0 . With the current decision thresholds, this rate tends to be very low but could become inflated with more liberal criteria for accepting a null or meaningless effect.

One final further way that the false positive rate could be reduced, which we do not cover here, would be to set a much tighter and more conservative prior on the underlying effect size (e.g., by making the priors on the groupgroup.2 parameter and sigma much more peaked around 0 and 1, respectively). Effect size estimates would thus be shrunk towards 0 and data that is more convincingly not a null effect would be needed to tip the posterior towards a positive conclusion.

\section{Misleading evidence in sequential analyses}

When taken literally as a binary decision to 'accept' or 'reject' that a parameter is above, below, or within some threshold or set of thresholds, certain decisions in Bayesian estimation become akin to hypothesis tests and can be subject to decision errors. Briefly, this is because decisions of the kinds above can be influenced by chance presentation of an observation or cluster of observations at extremes, which may skew the estimate. When a sequential analysis is performed, repeated checking of the data increases the number of opportunities we have to 'catch' the parameter after some such run of values. It is then possible that we conclude the underlying parameter is greater or smaller than it really is. This is a general phenomenon that is not specific to Bayesian parameter estimation: it can occur with frequentist hypothesis testing, Bayesian hypothesis testing, or frequentist parameter estimation when decision thresholds are used that are sensitive to such fluctuations in the incoming data (Kruschke, 2014, 2018).

This is one of the reasons that some authors have argued to aim for a certain level of precision in estimation as opposed to making decisions based upon the sizes of effects (Kruschke \& Liddell, 2018; Maxwell et al., 2008). Using simulations, Kruschke (2014) has shown how aiming for precision is not subject to the issues above, and this also makes sense conceptually: all else being equal, the precision of posterior estimates are largely controlled 
by the sample size and not so much by extreme values. The approach we present is perfectly compatible with aiming for precision. However, Kruschke's simulations also showed that the sample size requirements when stopping for precision can be rather large - often larger than when stopping on the basis of being able to accept or reject a parameter estimate. As we have shown, with the current approach it is possible to flexibly consider different criteria for stopping a sequential analysis so as to rein in the rates of misleading evidence, which can reduce issues associated with such stopping criteria. Our intention is not to advocate for any one approach or another, but rather to make the reader aware of the different possibilities and their associated costs.

\section{Summary of approach to power}

The approach to power analysis presented here involves 4 primary steps. In Step 1, we seek to generate a large number of data sets for analysis. There are many ways these data sets can be produced at: explicitly choosing a distribution of effect sizes, using a posterior distribution of effect sizes from a previous analysis, directly generating data from a previous Bayesian regression model ${ }^{3}$, constructing hypothetical data and using that to generate a posterior distribution from which data can be simulated, or picking a set of effect sizes for sensitivity assessments. Notably, we also suggest that one might consider some sort of 'null' or 'uninteresting' data set, which can be used to assess false positive rates. In addition, one need not focus on an effect size - group means or indeed any other parameter of interest could be selected. The key point is the ability to generate a sizable number of data sets to represent the phenomenon of interest.

Step 2 is to run many analyses of the data efficiently. This is the most time-consuming step of the process, and the time burden is eased by running analyses in parallel. The data should be analyzed using multiple possible sample sizes so that our ability to reach

\footnotetext{
${ }^{3}$ Generating new data from regression models can be achieved with the add_predicted_draws() function in tidybayes
} 
conclusions given various decision criteria can be assessed at different sample sizes. In practice, it will often be useful to initially run a more restricted number of regressions so that a rudimentary power analysis can be performed to help determine possible sample sizes to run more fully. Rather than directly extracting information about whether decision criteria have been met from these analyses and returning that information, the full posterior distribution can be returned from each analysis, resulting in a large but highly flexible set of data for analysis.

Step 3 is to summarize the posterior distributions returned in Step 2 to determine the proportion of times different decision criteria are met. As noted, the goal of the present paper is not to advocate any particular decision criterion - many criteria can be flexibly considered, so long as they are appropriate/relevant for the purpose the researcher intends. This stage of the analysis shows the likelihood of achieving a range of goals given various sample sizes, and can also display rates of misleading evidence, in fixed sample size analyses.

Step 4 is to run the posterior summaries through a function that can determine power and rates of misleading evidence when the data is analyzed sequentially. Again here, decision criteria can be flexibly considered with reference to the likelihood of achieving one's goals and the possible accumulation of misleading evidence. If such rates are unacceptable, more or less stringent criteria can be put through Step 3 and then passed to Step 4. In addition, the user can experiment with different stopping sequences to see if this rectifies any issues with the rates of goal achievement/misleading evidence. Note that this flexibility is intended for the planning stage, but rates of misleading evidence are not controlled appropriately if decision criteria are modified on the basis of seeing interim results (unless the probabilities of these decisions are also incorporated into the assessments, which is fraught with difficulty).

As far as we are aware, this proposed process is more flexible and efficient than previous efforts to incorporate power analysis into Bayesian estimation analyses. In the sections that follow, we conclude with some limitations and possible extensions to the approach. 


\section{Limitations and extensions}

The approach covered here and similar treatments of power from a Bayesian estimation perspective are limited by their relative difficulty and complexity in comparison to out-of-the-box software that is increasingly available for other analytic approaches. This limitation should be balanced with appreciation of the flexibility of the approach: with knowledge of different regression models, the broad approach can be extended to a range of models, including ordinal models, multilevel models, beta regression etc., as well as to a host of custom decision criteria. The time it can take to run the large number of regressions required can also be frustrating, even when run in parallel - especially when we are used to rapid results from other approaches (still, many thousands of regressions are being run in a matter of minutes, and one hopes that these analyses might help reduce time costs in the longterm). The reader is encouraged to experiment with less time-intensive simulations/regressions at first (e.g., try using fewer iterations, requesting fewer sample size steps) to get results more quickly and get a feel for different results before committing to a very large run of regressions.

One way in which the current approach may be extended to become 'more fully Bayesian' would be to not simply summarize the rates of goal achievement with point estimates, but to run a full analysis on the rates at which different goals are achieved as a Bayesian regression model. Going to this step was beyond the scope of this article, which may already be introducing readers to some novel approaches - we did not intend to further add to the complexity by also explaining another type of Bayesian analysis on the outcome data. However, it would be possible to run regression models on the rates at which different conclusions are reached, using different sample sizes, effect sizes, stopping criteria, analytic approaches (fixed vs. sequential), and checking sequences, to generate posterior estimates of the rates, fully encompassing the uncertainty of such estimates. This could have the advantage beyond simply an arbitrary preference for being 'more Bayesian': in principle the regression 
models might be used to predict rates given different sample sizes, effect sizes, and so on, without needing to run full, time consuming simulations at all the different combinations of these. Assessing the reliability of such estimates relative to brute simulation is a subject we are looking into further, as it may help reduce some of the time demands of these analyses.

More generally, Bayesian models are often subject to criticism or concern on the basis of the use of priors, which can impact upon the inferences drawn. The influence of priors on inferences may or may not be desirable - it depends on intent. In fact, priors might even be leveraged to reduce false positive rates. However, it can be informative to investigate different prior specifications to understand and show others if and how the priors have an influence. Whatever the case, information on what priors are used in analyses should be transparently presented.

\section{Consideration of psychological or other non-statistical factors in sequential analysis}

Researchers would be wise to keep in mind that taking care of statistical aspects of study design does not secure the study's success or integrity. A range of psychological factors may jeopardize even properly-powered studies (Elsey et al., 2021). In sequential analysis, one crucial assumption is that the data-generating process remains constant across the experimental cycle. However, if researchers observe the results at midway points, this may affect their behavior, in turn influencing participants with whom they interact. Researchers are advised to carefully consider how knowledge of interim effects might impact upon the datagenerating process, including researchers consciously or unconsciously pushing participants in a particular direction, but also simply affecting the motivation and diligence with which the researcher engages with participants. Various means of insulating sequential analyses from the possible psychological impact of interim data checks might be pursued (Elsey et al., 2021). Possibilities include automating analyses such that only information about whether to cease or continue data collection is presented to researchers (Beffara-Bret et al., 2019), or involving analysts who remain independent of the researchers who interact with 
participants/patients, in a similar vein to data monitoring committees often used in medical trials (Friedman et al., 2015; Lakens, 2014).

A final consideration is that researchers can be overly optimistic in assuming that if they do perform a sequential analysis, they are sure to be able to stop their data collection earlier than the maximum sample size. However, researchers may overestimate effect sizes when planning studies, or may simply be unlucky in their search for an effect, such that data collection continues for just as long as if a typical fixed $\mathrm{N}$ design were used. From experience with sequential analysis in practice, the author would recommend researchers truly entertain the maximum sample size as possible or even likely to be required, and consider the option to stop early as a bonus. Researchers are free to cease data collection owing to time or financial constraints if studies drag on or become more costly than anticipated, but there is little use in presenting a highly powered design that one is not really prepared to take to completion in the first place. Planning to stop at early stages is likely to produce frustration among the research team if the study repeatedly continues after possible stopping points. This is not only unpleasant, but could also affect how they interact with participants.

\section{Conclusion}

We have presented a general process with which power analyses can be performed in Bayesian estimation/Bayesian new statistics analyses. The 4-step framework allows assessment of both fixed $N$ and sequential designs, gives researchers the flexibility to consider a range of decision criteria, and can incorporate assessments of the rate of misleading evidence. By leveraging the power of parallel processing, the approach is also relatively fast. It is hoped that researchers can utilize and build upon the framework provided to devise wellpowered studies into important psychological phenomena. 


\section{Bibliography}

Anderson, S. F., Kelley, K., \& Maxwell, S. E. (2017). Sample-size planning for more accurate statistical power: A method adjusting sample effect sizes for publication bias and uncertainty. Psychological Science, 28(11), 1547-1562. https://doi.org/10.1177/0956797617723724

Bates, D., Sarkar, D., Bates, M. D., \& Matrix, L. (2007). lme4.

Beffara-Bret, B., Beffara-Bret, A., \& Nalborczyk, L. (2019). A fully automated, transparent, reproducible and blind protocol for sequential analyses. PsyArXiv. https://doi.org/10.31234/osf.io/v7xpg

Bürkner, P.-C. (2017). brms: An R package for Bayesian multilevel models using Stan. Journal of Statistical Software, 80(1), 1-28.

Cumming, G. (2014). The new statistics: Why and how. Psychological Science, 25(1), 7-29. https://doi.org/10.1177/0956797613504966

Elsey, J. W. B., Filmer, A. I., \& Stemerding, L. E. (2021). Don't forget the psychology in psychological analyses: The case of sequential testing. Psyarxiv. https://doi.org/10.31234/osf.io/tyqha

Friedman, L. M., Furberg, C. D., DeMets, D. L., Reboussin, D. M., \& Granger, C. B. (2015). Monitoring committee structure and function. In Funcdamentals of Clinical Trials (5th ed., pp. 343-372). Springer.

Ioannidis, J. P. A. (2005). Why most published research findings are false. PLoS Medicine, 2(8), e124. https://doi.org/10.1371/journal.pmed.0020124

Kay, M. (2020). Tidybayes (2.3.1).

Kruschke, J. K. (2013). Bayesian Estimation Supersedes the t Test. Journal of Experimental Psychology: General, 142(2), 573-603. https://doi.org/10.1037/a0029146

Kruschke, J. K. (2014). Goals, power, and sample size. In Doing Bayesian Data Analysis: A Tutorial with R, JAGS, and Stain (2nd ed., pp. 359-398). Academic Press. 
Kruschke, J. K. (2018). Rejecting or accepting parameter values in Bayesian estimation. Advances in Methods and Practices in Psychological Science, 1(2), 270-280. https://journals.sagepub.com/doi/abs/10.1177/2515245918771304

Kruschke, J. K., \& Liddell, T. M. (2018). The Bayesian New Statistics: Hypothesis testing, estimation, meta-analysis, and power analysis from a Bayesian perspective. Psychonomic Bulletin and Review, 25(1), 178-206. https://doi.org/10.3758/s13423-016-1221-4

Lakens, D. (2014). Performing high-powered studies efficiently with sequential analyses. European Journal of Social Psychology, 44(7), 701-710.

https://doi.org/10.1002/ejsp.2023

Lakens, D. (2021). Sample Size Justification. Psyarxiv. https://doi.org/https://doi.org/10.31234/osf.io/9d3yf

Lamberink, H. J., Otte, W. M., Sinke, M. R. T., Lakens, D., Glasziou, P. P., Tijdink, J. K., \& Vinkers, C. H. (2018). Statistical power of clinical trials increased while effect size remained stable: an empirical analysis of 136,212 clinical trials between 1975 and 2014. Journal of Clinical Epidemiology, 102, 123-128. https://www.sciencedirect.com/science/article/pii/S0895435618301409?casa_token=Wg UN_Ic0w9wAAAAA:ilwgLL3UkQAqn9xECQgv7aReUsRSrmDtnEohZCnvjy8iULBk8 POGYnVbo0LbmwRZzUfFqw-n

Maxwell, S. E., Kelley, K., \& Rausch, J. R. (2008). Sample size planning for statistical power and accuracy in parameter estimation. In Annual Review of Psychology (Vol. 59, pp. 537-563). https://doi.org/10.1146/annurev.psych.59.103006.093735

Schnuerch, M., \& Erdfelder, E. (2020). Controlling Decision Errors With Minimal Costs: The Sequential Probability Ratio t Test. Psychological Methods, 25(2), 206-226. https://doi.org/10.1037/met0000234

Schönbrodt, F. D., \& Stefan, A. M. (2018). BFDA: An R package for Bayes factor design analysis (0.4.0). 
Schönbrodt, F. D., \& Wagenmakers, E. J. (2018). Bayes factor design analysis: Planning for compelling evidence. Psychonomic Bulletin and Review, 25(1), 128-142. https://doi.org/10.3758/s13423-017-1230-y

Szucs, D., \& Ioannidis, J. P. A. (2017). Empirical assessment of published effect sizes and power in the recent cognitive neuroscience and psychology literature. PLoS Biology, 15(3). https://doi.org/10.1371/journal.pbio.2000797

Vaughan, D., Dancho, M., \& RStudio. (2021). furrr v0.2.2.

Wald, A. (1945). Sequential tests of statistical hypotheses. The Annals of Mathematical Statistics, 16(2), 117-186.

Wickham, H., Averick, M., Bryan, J., Chang, W., McGowan, L D A François, R., \& Yutani, H. (2019). Welcome to the Tidyverse. Journal of Open Source Software, 4(43), 1686. 